\title{
Being a foot and ankle surgeon in Italy in the era of COVID-19
}

\author{
Federico Giuseppe Usuelli ${ }^{1} \cdot$ Riccardo $^{\prime}$ Ambrosi $^{2}{ }^{\circledR}$
}

Received: 28 March 2020 / Accepted: 21 April 2020 / Published online: 23 April 2020

(c) European Society of Sports Traumatology, Knee Surgery, Arthroscopy (ESSKA) 2020

\section{Introduction}

On December 31, 2019, aggregate cases of an apparently new respiratory syndrome were reported to the World Health Organization (WHO) by Chinese national health authorities in the city of Wuhan [1]. Subsequently, the first two cases detected in Italy were on January 29, 2020, when a couple from the city of Wuhan travelling to Italy were admitted to the Lazzaro Spallanzani National Institute of Infectious Diseases, in Rome, after becoming ill and presenting with respiratory tract symptoms and fever. The respiratory samples test confirmed infection with SARS-COV2 [4].

The coronaviruses (CoVs) can be considered the major pathogens of emerging respiratory disease outbreaks. They are a large family of single-stranded RNA viruses that can be isolated in different animal species. Moreover, for reasons yet to be explained, these viruses can cross species barriers and can cause, in humans, illnesses ranging from the common cold to more severe diseases such as MERS and SARS [6].

This virus has grown, causing a pandemic and endangering global health [2]. To date (28 March, 2020) Italy has 70,065 positives, of which 10,023 have died. Particularly in Lombardy, the account of positive patients is 24,509 , of which 12,471 patients are hospitalized and 1319 in intensive care (https://opendatadpc.maps.arcgis.com/apps/opsdashboa rd/index.html\#/dae18c330e8e4093bb090ab0aa2b4892).

This worrying and alarming data has led the Italian government to take drastic measures - as never before-limiting exits, closing meeting points such as bars and restaurants and redistributing the intensive care access, thus limiting

Riccardo D’Ambrosi

riccardo.dambrosi@hotmail.it

Federico Giuseppe Usuelli

fusuelli@gmail.com

Humanitas San Pio X, Milan, Italy

2 IRCCS Istituto Ortopedico Galeazzi, Via Galeazzi 4, 20161 Milan, Italy elective surgery. This situation directly involves daily lives of everyone. Hence, it also has a significant impact on orthopedic surgery and the practice of foot and ankle specialist surgeons.

Although foot and ankle surgeons do not play a key role in the treatment of COVID-19 patients, efforts need to be made to continue some activities while respecting three important principles: being responsible citizens while respecting current government regulations and restrictions; being effective communicators and using the medical-patient relationship in supporting and emphasizing the restriction regulations to our patients (i.e. https://www.youtube.com/watch?v=aIL10 cEUIhI); and acting as care providers for patients under treatment or in need with urgent foot and ankle conditions.

In this scenario, orthopedic foot and ankle surgeons continue to provide their care to mainly three different groups of patients:

- Post-operative.

- Scheduled for elective surgery.

- Traumatology/emergency.

\section{Post-operative patients: care and coaching}

This category includes patients who have undergone surgery within 6 months prior to the outbreak of COVID-19 cases.

It is mandatory to minimize the access of post-operative patients to reference Centers to reduce the risk of the COVID-19 infection. As such, small centers or specialized offices are preferred. In the event that imaging technology is not available in small offices, this analysis should be postponed if not urgent. Furthermore, the lead surgeon can successfully focus on the referral of patients for local care, using the link with former fellows and colleagues that practice on country sites, promoting scientific and clinical exchange, to reduce the need for traveling and visiting of larger reference centers, thereby decreasing risk of contamination. The importance of the mentor-trainee relationship was highlighted by Schiller and DiGiovanni who argued that the relationship established between a 
resident and their foot and ankle mentor appears to have the greatest impact on pursuing foot and ankle surgery as a career [10]. Accordingly, this interpersonal connection can be used to foster care and trust, maintain patient care and minimize the risk of patient's contamination and spread of the COVID-19 outbreak.

For longer follow-up consultation, the advent of technologies and telemedicine has made everything easier. At the moment, user-friendly apps, such as "iMeApp", and other software allow patients to easily make available relevant clinical information to their care-provider before a formal telemedicine appointment.

The "iMeApp" allows patients to provide clinical information and update their surgeon following a three-step process:

Step 1. Taking clinical photos of the wound/condition.

Up to five clinical pictures can be uploaded, which are simple photos with a frame that is as "clear" and "clean" as possible to facilitate interpretation of the images. Technically, they are images of the foot under standing upright load: monopodalic and bipodalic support, with a fifth in which the surgical wound is also depicted.

Step 2. Recording a video.

The patient records a short video while walking in a straight line, allowing the interpretation of progressive weight-bearing protocols and gait pattern.

Step 3. Photograph or scanning important documents.

The patient can upload scanned documents to facilitate and expedite a formal telemedicine appointment, including official identification documents, surgical/discharge reports and referral documents.

The use of similar apps or software to optimize connection between healthcare providers and providers and patients has already been reported in the literature. An article by Giordano et al. demonstrated how WhatsApp Messenger (Facebook Inc.-Mountain View, California, United States of America) represents a promising system, whether used as a communication tool between health care professionals, health care professionals and the general public, or as a learning tool for providing health care information to professionals, patients or the general population [3].

There are some concerns about security and privacy issues associated with the use of Whats App. However, until now, there is no scientific evidence that patient information sent to the consultant via a secure messaging application reached the other person and this concerns is only a prediction [5].

Therefore, when selecting a telemedicine system, it is mandatory to respect the General Data Protection Regulation (GDPR $\left.n^{\circ} 2016 / 679\right)$ that provides data privacy and security provisions for safeguarding medical information. The protection of personal and contact data should be protected with passwords.
All these procedures allow providers to monitor patients from a distance, reducing the risk of complications, such as infections or wound dehiscence; in case of complications, it is easier to proceed with referral for in person visit, treatment and possible hospitalization.

This has also been confirmed by a recent study by Rosner et al. in which the authors demonstrated that patients enrolled on an automated digital patient engagement platform after hip and knee arthroplasties demonstrated a significant reduction in potentially avoidable 90 -day costs, a $45.4 \%$ non-significant relative reduction in 90-day hospital admissions and a $54.4 \%$ significant relative reduction in 90-day complications [9]. Furthermore, while having continued treatment, even at distance, patients do not feel abandoned and data collection is optimized as emphasized by Premkumar et al. [7]. The authors evaluated whether a novel mobile phone short message service platform could lead to better patient response rate to surveys on pain and opioid use. They concluded that the application of widely available technology can improve the measurement of post-operative patient-reported outcomes.

\section{Elective surgery: what are the limits?}

\section{When to limit elective surgery (phase 1)}

In this phase, the main critical issue is availability of Intensive Care Units.

Two main criteria must lead to the reduction of elective surgery:

- Not impacting the number of beds in intensive care (careful selection of the elective patients).

- Not being exposed to higher risks patients in critical clinical conditions.

If needed, the procedures should be ideally organized in a COVID-19-free hospital or a dedicated COVID-19 free area in a general hospital, respecting local and national regulations. During a possible phase of restructuring and reorganization the system to allow elective procedures, it is mandatory to create mechanisms in which patients and medical personnel are screened, evaluating for presence of fever or symptoms such as cough and cold.

Furthermore, the hospital may become a vector of infection. Therefore, it is critical to reduce the affluence to the hospital. As such, it is recommended to reduce elective inand out-patient procedures, to avoid crowded ambulatory surgical centers.

In any case, priority should be given to patients with a high level of disability, with absence of important comorbidities and in good enough conditions to presumably exclude the need of intensive care. 
Obviously, not to overload the intensive care dedicated to COVID-19, in this phase, we currently operate only selected patients, in particular those with characteristics that respect the following criteria: 65 years-old or younger, absence of diabetes, respiratory and cardiovascular conditions and hemoglobin higher than 13.5.g/dL. All these measures are to prevent complications; about $15-20 \%$ of suspected and confirmed patients with SARS-CoV-2 infection in fever clinics developed severe hypoxemia (from the second week of disease course), and require some form of ventilatory support such as high-flow nasal cannula, and non-invasive and invasive mechanical ventilation. In addition, other complications might occur, including shock, acute kidney injury, gastrointestinal bleeding and rhabdomyolysis [8].

\section{When to postpone elective surgery (phase 2)}

In this phase, we have to face further critical issues: bed availabilities and highly qualified health-care providers (anesthesiologists and nurses).

Elective surgeries should be postponed during the time of the health care crisis for two main reasons:

- Keeping as many free beds as possible for COVID-19 patients.

- Focusing health care resources on the treatment of the pandemic, in particular anesthetist staff.

A different evolution of the pandemic may motivate different choices such as in Korea where according to the same criteria, there is no need to postpone elective surgery [11].

\section{Trauma and emergency cases}

An effective plan could be to keep a number of hospitals COVID-19 free to perform elective surgery and traumatology.

Due to the effectiveness of the infection, this was not the plan for Lombardy. In our region, COVID-19 free areas have been created within hospitals to continue with trauma surgery. We are treating these patients in COVID-19 free areas but with the same protocols used for positive COVID-19 patients (e.g. single rooms, denied visits).

The path currently being followed is shown in the following table.

\begin{tabular}{llll}
\hline & $\begin{array}{l}\text { COVID-19 free } \\
\text { hospital }\end{array}$ & $\begin{array}{l}\text { COVID-19 } \\
\text { free area in } \\
\text { general hos- } \\
\text { pital }\end{array}$ & $\begin{array}{l}\text { COVID-19 dedi- } \\
\text { cated hospital }\end{array}$ \\
\hline $\begin{array}{l}\text { Type of sur- } \\
\text { gery }\end{array}$ & $\begin{array}{c}\text { Postpone elec- } \\
\text { tive surgery } \\
\text { Trauma sur- } \\
\text { gery }\end{array}$ & $\begin{array}{l}\text { Trauma sur- } \\
\text { gery }\end{array}$ & No surgery \\
& & \\
\hline
\end{tabular}

\section{Conclusions}

COVID-19 has proven to be a serious disease that has managed to completely change our daily lives.

Anything planned before a pandemic may seem alarmist but everything we do after will seem inadequate. Therefore, authorities should not be worried to implement lockdowns. Moreover, politicians should take prompt decisions and doctors should be involved to spread the message, supported by their positive influence on people and patients. Our role as orthopedic foot and ankle surgeons must primarily be to act as an example and respect the rules; after this, it is essential to follow our patients up as far as possible, promoting healthy behavior and developing alternative systems to provide continuous care and minimizing hospital access. Moreover, great attention should be given to creating either a COVID-19-free hospital or a hospital area, to proceed with safe selected elective procedures as well as urgent and traumatic cases.

Acknowledgements A heartfelt thanks to all medical and paramedical staff, nurses and volunteers from the medical and civil protection sector and all operators who are experiencing a particularly difficult period due to the health emergency linked to COVID-19.

\section{Compliance with ethical standards}

Conflict of Interest Dr. Usuelli reports the following conflict of interest outside the submitted work:ZimmerBiomet Consultant; Lima Consultant; Geistlich Consultant; Arthrex Consultant.

Funding None.

Ethical approval This article does not contain any studies with human participants performed by any of the authors.

\section{References}

1. Adhikari SP, Meng S, Wu YJ, Mao YP, Ye RX, Wang QZ, Sun C, Sylvia S, Rozelle S, Raat H, Zhou H (2020) Epidemiology, causes, clinical manifestation and diagnosis, prevention and control of coronavirus disease (COVID-19) during the early outbreak period: a scoping review. Infect Dis Poverty 9:29. https://doi.org/10.1186/ s40249-020-00646-x 
2. Albarello F, Pianura E, Di Stefano F, Cristofaro M, Petrone A, Marchioni L, Palazzolo C, Schininà V, Nicastri E, Petrosillo N, Campioni P, Eskild P, Zumla A, Ippolito G, COVID 19 INMI Study Group (2020) 2019-novel Coronavirus severe adult respiratory distress syndrome in two cases in Italy: an uncommon radiological presentation. Int J Infect Dis 93:192-197. https://doi. org/10.1016/j.ijid.2020.02.043

3. Giordano V, Koch H, Godoy-Santos A, Dias Belangero W, Esteves Santos Pires R, Labronici P (2017) WhatsApp messenger as an adjunctive tool for telemedicine: an overview. Interact J Med Res 6:e11. https://doi.org/10.2196/ijmr.6214

4. Giovanetti M, Benvenuto D, Angeletti S, Ciccozzi M (2020) The first two cases of 2019-nCoV in Italy: where they come from? J Med Virol 92:518-521. https://doi.org/10.1002/jmv.25699

5. Johnston M, King D, Darzi A (2016) Reply to the letter: WhatsApp with patient data transmitted via instant messaging? Am J Surg 211:301-302. https://doi.org/10.1016/j.amjsurg.2015.04.004

6. Perrella A, Carannante N, Berretta M, Rinaldi M, Maturo N, Rinaldi L (2020) Novel Coronavirus 2019 (Sars-CoV2): a global emergency that needs new approaches. Eur Rev Med Pharmacol Sci 24(4):2162-2164. https://doi.org/10.26355/eurrev_20200 2_20396

7. Premkumar A, Lovecchio FC, Stepan JG, Kahlenberg CA, Blevins JL, Albert TJ, Cross MB (2019) A novel mobile phone text messaging platform improves collection of patient-reported post-operative pain and opioid use following orthopedic surgery. HSS J 15(1):37-41. https://doi.org/10.1007/s11420-018-9635-3

8. Qiu H, Tong Z, Ma P, Hu M, Peng Z, Wu W, Du B, China Critical Care Clinical Trials Group (CCCCTG) (2020) Intensive care during the coronavirus epidemic. Intensive Care Med. https://doi. org/10.1007/s00134-020-05966-y

9. Rosner BI, Gottlieb M, Anderson WN (2018) Effectiveness of an automated digital remote guidance and telemonitoring platform on costs, readmissions, and complications after hip and knee arthroplasties. J Arthroplasty 33:988-996. https://doi.org/10.1016/j. arth.2017.11.036

10. Schiller JR, DiGiovanni CW (2008) Foot and ankle fellowship training: a national survey of past, present, and prospective fellows. Foot Ankle Int 29:34-41. https://doi.org/10.3113/ FAI.2008.0034

11. Zastrow M (2020) South Korea is reporting intimate details of COVID-19 cases: has it helped? Nature. https://doi.org/10.1038/ d41586-020-00740-y

Publisher's Note Springer Nature remains neutral with regard to jurisdictional claims in published maps and institutional affiliations. 